\title{
Analysis of the Effect of Sampling Site of Umbilical Artery Doppler on the Variability of Umbilical Artery Pulsatility Index
}

\author{
Original \\ Mohammad Ayyad ${ }^{1}$,Marwa Romeih', Karim El-Etriby ${ }^{3}$, Yasser Abou Talib ${ }^{4}$ \\ Article \\ ${ }^{1,3}$ Department of Obstetrics and Gynecology, ${ }^{2}$ Department of Radiodiagnosis, Faculty of \\ Medicine, Helwan University, ${ }^{4}$ Department of Obstetrics and Gynecology, Faculty of Medicine, \\ Ain-Shams University, Cairo, Egypt
}

\begin{abstract}
Background: Analysis of the umbilical artery (UA) flow by means of pulsed Doppler has become a standard for antenatal care of high-risk pregnancies.

Aim: The aim of the present study was to test the hypothesis that standardizing the site of sampling of umbilical artery Doppler reduces the variability of umbilical artery Pulsatility Index (PI).

Materials and Methods: The present study was Prospective study included 120 pregnant women - with singleton fetuses of 28 week of gestation till term and delivery-attending outpatient clinic at Helwan University Hospitals. All pregnant females were followed up monthly minimum for 3 occasions till delivery.

Results: The present study revealed the presence of significant variability in the measurements of umbilical artery PI among the sampling sites as well as among the different time points at which the measurements were taken, with poor repeatability and reproducibility of the umbilical artery PI measurements. Variability in umbilical artery PI among the sampling sites was demonstrated in our study. There were significant differences in the mean umbilical artery PI among the four sampling sites, with higher mean umbilical artery PI at PVC site, followed by AI, FL and PL sites. Intraclass correlation was computed to test reliability, showing poor agreement among the four sampling sites. These findings suggest that standardization of the site of sampling may reduce variability and increase reliability of measurements.

Conclusion:Standardization of the site of sampling of umbilical artery Doppler may reduce variability and increase reliability of measurements of umbilical artery Pulsatility Index.
\end{abstract}

Key Words: Sampling site, umbilical artery Doppler, umbilical artery pulsatility index, variability

Received: 01 September 2020, Accepted: 10 October 2020

Corresponding Author: Marwa Romeih, Department of Radiodiagnosis, Faculty of Medicine, Helwan University, Tel.: +201273195969, E-mail: marwaromeih@gmail.com

ISSN: 2090-7265, August 2021, Vol.11, No. 3

\section{INTRODUCTION}

Analysis of the umbilical artery (UA) flow by means of pulsed Doppler has become a standard for antenatal care of high-risk pregnancies such as growth-restriction, multiple pregnancy, pregnancy-induced hypertension or oligohydramnios ${ }^{[1]}$.

There are four potential sites for insonation of the umbilical artery placental insertion (PL), free loop (FL) of cord, abdominal insertion (AI), and intraabdominal (perivesical) $\left(\mathrm{PVC}^{[2]}\right.$.

The ratios (indices) of the resistance to blood flow (impedance) are calculated using the following formulas: $\mathrm{S} / \mathrm{D}$ ratio $=$ (systolic / diastolic ratio). Resistance index $(\mathrm{RI})=($ systolic velocity - diastolic velocity / systolic velocity). Pulsatility index $(\mathrm{PI})=$ (systolic velocity - diastolic velocity / mean velocity). In the normal fetus, the resistance to flow (impedance) decreases in the umbilical artery due to increased numbers of tertiary stem villi as the placenta matures ${ }^{[3]}$.

Umbilical artery Doppler assessment has been shown to reduce perinatal mortality and morbidity in high-risk obstetric situations ${ }^{[4]}$.

Placental insufficiency may be encountered in some cases, including intrauterine growth restriction, pregnancyinduced hypertension, oligohydramnios, and multiple pregnancies. However, the measured value of the umbilical artery PI differs depending on the site of measurement. Hence, it is important to consider the sampling site when abnormal measurements of the umbilical artery PI are obtained, particularly when the timing of delivery will be determined based on these measurements. Standardization 
of the sampling site could help clinicians by providing a reference point for measurements, allowing for consistent interpretations and valid comparisons of the obtained measurements ${ }^{[2]}$.

\section{AIM OF THE WORK}

The aim of the present study was to test the hypothesis that standardising the site of sampling of umbilical artery Doppler reduces the variability of umbilical artery Pulsatility Index (PI).

\section{PATIENTS AND METHODS}

This prospective study included 120 pregnant women with singleton fetuses of 28 week of gestation till term and delivery-attending outpatient clinic at Helwan University Hospitals for routine antenatal care. The duration of the study was one year started from July 2019 to July 2020.

Patients : The study includes 120 pregnant women attending outpatient clinic at Helwan University Hospitals.

Inclusion criteria : Singleton pregnancies.Gestational age between 28 and 41 weeks confirmed by US in the first trimester. Absence of sonographic evidence of fetal abnormalities. Normal fetal size $\left(>10^{\text {th }}\right.$ and $<90^{\text {th }}$ percentile according to standards).

Exclusion criteria : Intra Uterine Growth Restriction (IUGR) fetuses. Pregnant women with chronic diseases e.g. Hypertension and DM.

Ethics approval was obtained from Faculty of Medicine Helwan University, Research Ethics Committee and each pregnant woman gave an informed consent to partake in the study.

All ultrasound examinations were done by the same examiner to minimize interobserver variability in the outpatient clinic using advanced ultrasound machine (2015 GE Logiq P5 Premium BT11 OB/Vasc Ultrasound System) using convex sector probes (2-8 MHz) and standard obstetric settings. Ultrasound examinations were performed as standard of care. All pregnant females were followed monthly minimum for 3 occasions till delivery; at each visit the following will be done:

Women were requested to be in a semi-recumbent position with a slight lateral tilt. This minimizes the risk of developing supine hypotension syndrome due to caval compression.

At the beginning of the examination, the routine ultrasound examination of the fetus was done (biometry, assessment of placental location and amniotic fluid volume).
Doppler examinations should be conducted only during fetal apnea and in the absence of fetal hiccup, excessive movement or fetal arrhythmia, because there is a breathing related modulation of arterial pulsatility.

The Doppler waveforms were performed and the Doppler angle was maintained as close to 60 as possible to achieve reproducible results, sample gate size was adjusted to the target vessel. Pulse Repetition Frequency was modulated to prevent aliasing.

Doppler examination was done at four potential sites for insonation of the umbilical artery which are: placental insertion (PL), free loop (FL) of cord, abdominal insertion (AI), and intraabdominal (perivesical) (PVC).

The fetal bladder was identified in the coronal section by using gray scale. The color mode was then applied to identify the PVC arteries.

Doppler waveforms were obtained in the position where the vessels coursed along the side walls of the bladder. The gauge was placed over the vessel, ensuring that the sample size was large enough to allow all velocity waveforms to be interrogated. A right or left umbilical artery was been examined, no distinction was made between both of them.

Because it was difficult to clearly identify the two arteries at the other sites, only one vessel was interrogated. The PI will be measured using the automatic trace function.

Two readings of PI were taken from each site and the average of both values was taken. The procedure was repeated for the AI, FL, and PL. Each study lasted about 10-20 min. Comparison of the four readings of the PI will be done between baseline and follow up values.

The outcome details were assessed by recording the neonatal mortality and morbidity, prematurity and neonatal measurements. Outcome details were collected either from hospital records, or by contacting the participants on the telephone.

Statistical analyses of the results were done using the appropriate statistical tests.

\section{RESULTS}

Demographics data of the baby's gestational age at every scan, time of delivery as well as the birth weight. Satisfactory flow velocity waveforms were obtained successfully from all fetuses at each one of four sampling site (FL, PL, PVC and AI), in every scan of the three scans between 28 and 40 weeks (Table 1).

Median gestational age in $1^{\text {st }}$ scan was 28 weeks 
and 32 weeks at $2^{\text {nd }}$ scan and 36 weeks at $3^{\text {rd }}$ scan. Median gestational age at delivery was 39 weeks and 3100 gm birth weight. PI was measures in four sites and correlated with gestational age (Table 2).

Umbilical artery PI was significantly negatively correlated with the gestational age (i.e. the PI was decreased by increasing GA) at $1^{\text {st }}$ scan at the four site of examination FL.PL.PVC, AI sites. ( $r=-0.251$ for PVC and $P<0.006)$. At the $2^{\text {nd }}$ scan, there was significant negative correlation between PI and GA only at the PVC site $(\mathrm{r}=-0.217, p<0.017)$. At $3^{\text {rd }}$ scan, there was significant negative correlation between PI and GA only at the AI site $(-0.252, p<0.006)$. We compared the umbilical artery PI measurements at the four sites in three different scan (Table 3).

At the $1^{\text {st }}$ scan, there were significant differences of the mean umbilical artery PI between the four sampling sites $(p<0.001)$. The mean umbilical artery PI was significantly higher at PVC site $(1.14 \pm .14)$ compared to AI, FL, and PL sites $(1.14 \pm .14,1.02 \pm .14,93 \pm .12$, respectively). Intraclass correlation coefficient revealed significant poor agreement between the 4 sites $(0.242, p<0.001)$.
At the $2^{\text {nd }}$ scan, there were significant differences of the mean umbilical artery PI between the four sampling sites $(p<0.001)$. The mean umbilical artery PI was significantly higher at PVC site $(1.02 \pm .12)$ compared to AI, FL, and PL sites $(.99 \pm .11,91 \pm .14,83 \pm .11$, respectively). Intraclass correlation coefficient revealed significant poor agreement between the 4 sites $(0.293, p<0.001)$

At the $3^{\text {rd }}$ visit, there were significant differences of the mean umbilical artery PI between the four sampling sites $(p<0.001)$.

The mean umbilical artery PI was significantly higher at PVC site $(.90 \pm .08)$ compared to AI, FL, and PL sites $.90 \pm .09, .78 \pm .10,69 \pm .13$, respectively). Intraclass correlation coefficient revealed significant poor agreement between the 4 sites $(0.283, p<0.001)$.

We compared the mean measurement at each single site in the three scans and the mean umbilical artery PI at FL site was significantly lower at visit 3 compared to visits 1 and 2 (Table 4 and Figure 1).

The mean umbilical artery PI at each site was significantly lower at visit 3 compared to visits 1 and 2 .

Table 1: Demographics data of the baby's gestational age at every scan, time of delivery as well as the birth weight

\begin{tabular}{|c|c|c|}
\hline \multirow{3}{*}{ GA at 1 st scan (weeks) } & Minimum- Maximum & $28.0-31.0$ \\
\hline & Mean \pm SD & $28.4 \pm .7$ \\
\hline & Median & 28.0 \\
\hline \multirow{4}{*}{ GA at 2nd scan (weeks) } & Minimum- Maximum & $32.0-35.0$ \\
\hline & Mean \pm SD & $32.5 \pm .7$ \\
\hline & Median & 32.0 \\
\hline & Minimum- Maximum & $36.0-39.0$ \\
\hline \multirow[t]{3}{*}{ GA at 3rd scan (weeks) } & Mean \pm SD & $36.7 \pm .9$ \\
\hline & Median & 37.0 \\
\hline & Minimum- Maximum & $37.0-41.0$ \\
\hline \multirow[t]{3}{*}{ GA at delivery (weeks) } & Mean \pm SD & $38.8 \pm 1.1$ \\
\hline & Median & 39.0 \\
\hline & Minimum- Maximum & $2700.0-4200.0$ \\
\hline \multirow[t]{2}{*}{ Birth weight (Grams) } & Mean \pm SD & $3192.5 \pm 316.7$ \\
\hline & Median & 3100.0 \\
\hline
\end{tabular}


Table 2: Correlation between gestational age and umbilical artery PI

\begin{tabular}{|c|c|c|c|c|c|c|}
\hline & \multicolumn{2}{|c|}{ GA at visit 1} & \multicolumn{2}{|c|}{ GA at visit 2} & \multicolumn{2}{|c|}{ GA at visit 3} \\
\hline & $\mathrm{r}$ & Pvalue & $\mathrm{R}$ & Pvalue & $\mathrm{r}$ & $P$ value \\
\hline FL & -0.273 & $0.003 *$ & 0.040 & 0.662 & -0.036 & 0.694 \\
\hline PL & -0.286 & $0.002 *$ & 0.133 & 0.149 & 0.057 & 0.538 \\
\hline PVC & -0.251 & $0.006^{*}$ & -0.217 & $0.017 *$ & -0.092 & 0.319 \\
\hline AI & -0.358 & $<0.001^{*}$ & -0.098 & 0.288 & -0.252 & $0.006^{*}$ \\
\hline
\end{tabular}

*significant at $p<0.05$

Table 3: Umbilical artery PI measurements at the four sites in the three scans

\begin{tabular}{|c|c|c|c|c|c|c|c|}
\hline & & \multicolumn{4}{|c|}{ Sampling site } & \multicolumn{2}{|c|}{ Repeated measure ANOVA } \\
\hline & & FL & PL & PVC & AI & $\mathrm{F}$ & $P$ value \\
\hline & $1^{\text {st }}$ scan & $1.02 \pm .14$ & $.93 \pm .12$ & $1.14 \pm .14$ & $1.14 \pm .14$ & 88.08 & $<0.001 *$ \\
\hline & $2^{\text {nd }}$ scan & $.91 \pm .14$ & $.83 \pm .11$ & $1.02 \pm .12$ & $.99 \pm .11$ & 103.67 & $<0.001^{*}$ \\
\hline & $3^{\text {rd }}$ scan & $.78 \pm .10$ & $.69 \pm .13$ & $.90 \pm .08$ & $.90 \pm .09$ & 279.85 & $<0.001^{*}$ \\
\hline \multicolumn{8}{|l|}{ Mean \pm SD } \\
\hline & \multicolumn{7}{|c|}{ Post hoc test (Bonferroni test) revealed significant different between all sites at each of the three scans $(p<0.001 *)$} \\
\hline & \multicolumn{7}{|c|}{ Type A Intraclass correlation coefficient (testing an absolute agreement between the 4 sites) } \\
\hline & \multicolumn{7}{|c|}{ At $1^{\text {st }} \operatorname{scan}=0.242,(95 \% \mathrm{CI}=0.101-0.385), p<0.001 *$} \\
\hline & \multicolumn{7}{|c|}{ At $2^{\text {nd }} \operatorname{scan}=0.293,(95 \% \mathrm{CI}=0.120-0.456), p<0.001 *$. } \\
\hline & \multicolumn{7}{|c|}{ At $3^{\text {rd }}$ scan $=0.283,(95 \% \mathrm{CI}=0.055-0.495), p<0.001^{*}$. } \\
\hline
\end{tabular}

*significant at $p<0.05$

Table 4: The mean PI at the four sites in the three scans

\begin{tabular}{|c|c|c|c|c|c|c|}
\hline & & \multicolumn{3}{|c|}{ Visits } & \multicolumn{2}{|c|}{ Repeated measure ANOVA } \\
\hline & & Visit 1 & Visit 2 & Visit 3 & $\mathrm{~F}$ & $P$ value \\
\hline \multirow[t]{4}{*}{ Mean \pm SD } & $\mathrm{FL}$ & $1.02 \pm .14$ & $.91 \pm .14$ & $.78 \pm .10$ & 202.05 & $<0.001 *$ \\
\hline & PL & $.93 \pm .12$ & $.83 \pm .11$ & $.69 \pm .13$ & 192.24 & $<0.001 *$ \\
\hline & PVC & $1.14 \pm .14$ & $1.02 \pm .12$ & $.90 \pm .08$ & 244.07 & $<0.001^{*}$ \\
\hline & AI & $1.10 \pm .15$ & $.99 \pm .11$ & $.90 \pm .09$ & 150.77 & $<0.001 *$ \\
\hline
\end{tabular}

Post hoc test (Bonferroni test) revealed significant difference between measurements at the three visits for each site $(p<0.001 *)$

*significant at $p<0.05$ 


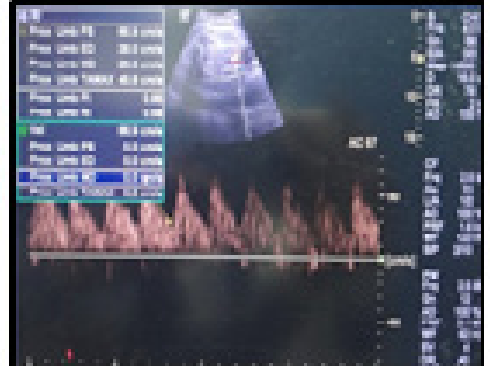

A

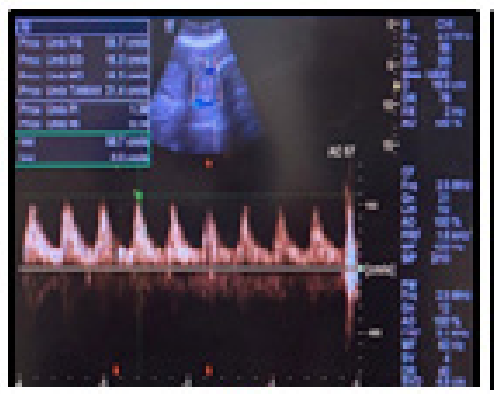

n

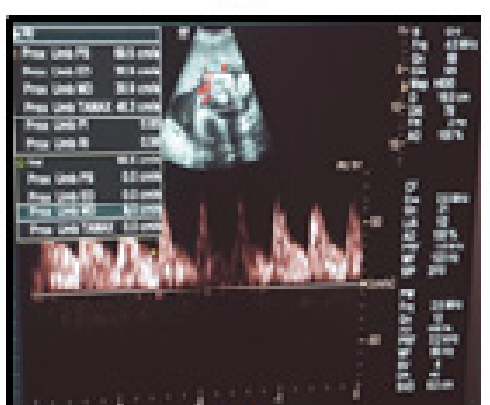

G

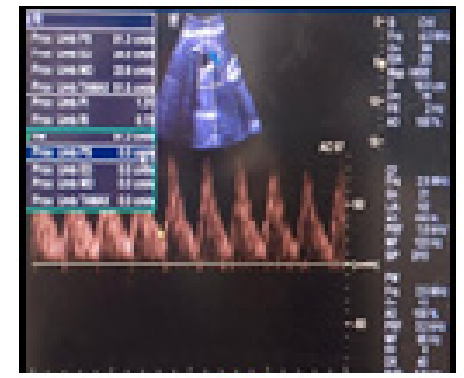

J

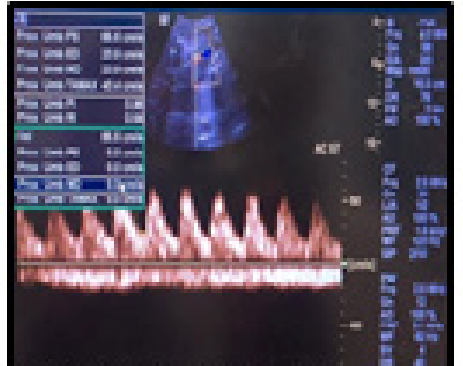

b

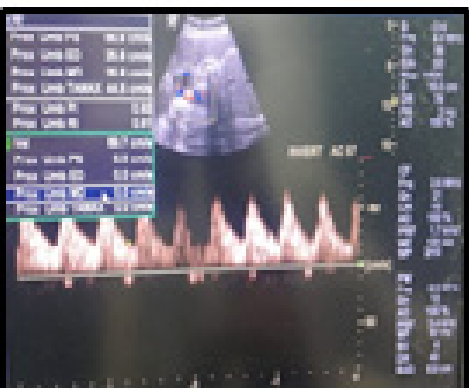

*

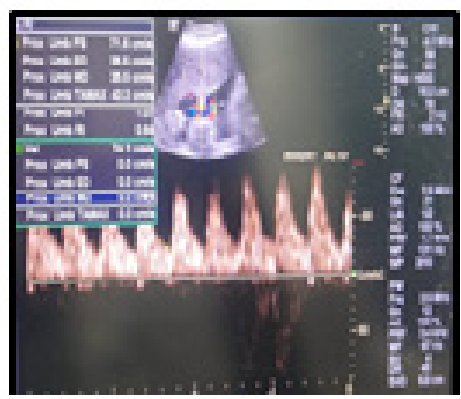

b

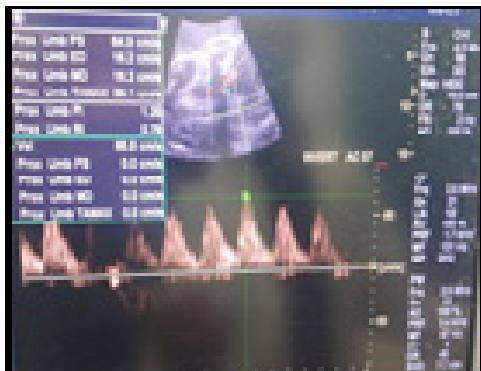

1.

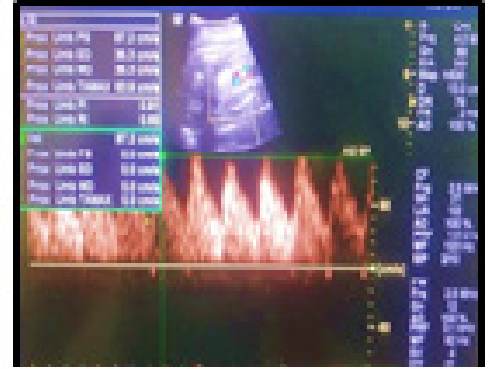

c
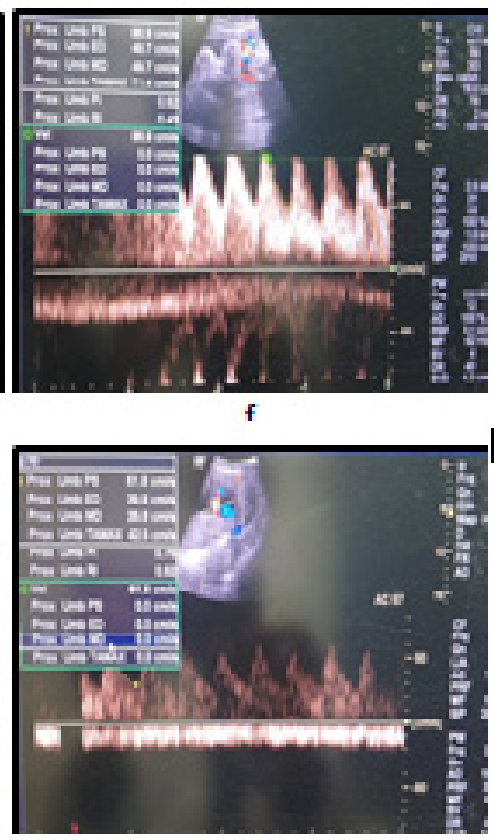

1

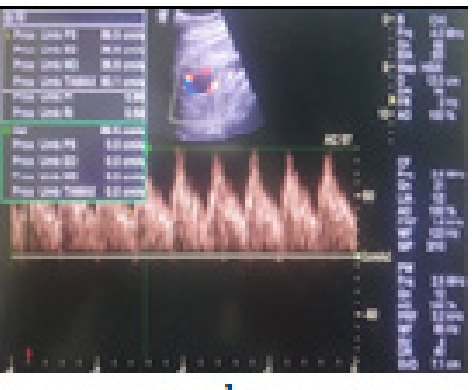

1

Fig. 1: Showing Umbilical artery Doppler assessment in the four potential sites of umbilical artery in a pregnant woman aged 30 year,G3P2; the Doppler exam performed in three occasions; the first scan was at $28^{\text {th }}$ week of gestation, the second scan was at $32^{\text {th }}$ week of gestation, the third scan was at $36^{\text {th }}$ week of gestation: Abdominal Insertion site (Al): a,b,c; Free loop site (FL): d,e,f; Placental End site (PL): g,h,i; Intrabdomenal or Perivesical site (PVC): $\mathrm{j}, \mathrm{k}, \mathrm{l}$. 


\section{DISCUSSION}

Analysis of the umbilical artery (UA) flow by means of pulsed Doppler has become a standard for antenatal care of high-risk pregnancies such as growth-restriction, multiple pregnancy, pregnancy-induced hypertension or oligohydramnios ${ }^{[1]}$.

The ratios (indices) of the resistance to blood flow (impedance) are calculated using the following formulas: $\mathrm{S} / \mathrm{D}$ ratio $=($ systolic $/$ diastolic ratio). Resistance index $(\mathrm{RI})=($ systolic velocity - diastolic velocity / systolic velocity). Pulsatility index $(\mathrm{PI})=$ (systolic velocity diastolic velocity / mean velocity). In the normal fetus, the resistance to flow (impedance) decreases in the umbilical artery due to increased numbers of tertiary stem villi as the placenta matures ${ }^{[3]}$.

Placental insufficiency may be encountered in some cases, including intrauterine growth restriction, pregnancyinduced hypertension, oligohydramnios, and multiple pregnancies. However, the measured value of the umbilical artery PI differs depending on the site of measurement. Hence, it is important to consider the sampling site when abnormal measurements of the umbilical artery PI are obtained, particularly when the timing of delivery will be determined based on these measurements ${ }^{[2]}$.

Standardization of the sampling site could help clinicians by providing a reference point for measurements, allowing for consistent interpretations and valid comparisons of the obtained measurements ${ }^{[2]}$

The aim of the current study was to test the hypothesis that standardizing the site of sampling of umbilical artery Doppler can reduce the variability of umbilical artery PI. We measured umbilical artery PI at four sites: PL, FL, AI, and PVC. The PL and AI sites represent fixed insertion of the umbilical cord into the placenta and the fetus, respectively. The FL site encompasses the non-fixed portion of the umbilical cord.

Previous research reported that the Doppler waveforms obtained from the fetal insertion end had higher sensitivity, while measurements obtained from the placental end had higher specificity ${ }^{[6,7,8]}$

We did not make a distinction between the right and left umbilical arteries. The Doppler waveforms were obtained from one umbilical artery, irrespective of laterality, which had the best position and angle for insonation at the time of sampling. The right and left umbilical arteries can be identified easily at the intra-abdominal portion but with difficulty at the other sites as both arteries originate from internal iliac arteries then each of them run downwards along the side wall of the fetal urinary bladder then both arteries form a cylindrical helix around the umbilical vein.
The results of the present study revealed the presence of significant variability in the measurements of umbilical artery PI among the sampling sites as well as among the different time points at which the measurements were taken, with poor repeatability and reproducibility of the umbilical artery PI measurements.

Variability in umbilical artery PI among the sampling sites was demonstrated in our study. There were significant differences in the mean umbilical artery PI among the four sampling sites, with higher mean umbilical artery PI at PVC site, followed by AI, FL, and PL sites. Intraclass correlation was computed to test reliability, showing poor agreement among the four sampling sites (correlation coefficient $=0.242, p<0.001$ )

These findings are in agreement with those of previous studies $^{[9,10,11,3,2,12]}$.

Mehalek et al. (1989) performed pulsed Doppler duplex sonography on 58 pregnant patients, measuring umbilical artery waveforms at the fetal abdomen and near the placental end of the cord. Their results showed that the mean PI was significantly higher at the fetal site than the placental site $(1.6 \pm 0.4$ and $\pm 1.1 \pm 0.2$, respectively, $p<0.01$ ). Similar significant differences were also observed in S/D and RI between the two sites ${ }^{[9]}$.

Sonesson et al. (1993) studied umbilical artery Doppler waveforms in 269 normal pregnancies. They concluded that the S/D ratio, PI, and RI were significantly higher at the fetal end of the cord compared to the measurements obtained at placental end, regardless of the gestational age $^{[10]}$.

Schaefer et al. (2000) performed Doppler examinations of umbilical artery PI in 48 normal pregnancies at four sites: the intra-abdominal portions of the right and the left umbilical arteries, the free loop, and the placental insertion. They found that the PI at the intra-abdominal segment was significantly higher than that at the free loop and at the placental insertion ${ }^{[11]}$.

Acharya et al. (2005) have taken repeated measurements of the umbilical artery indices in 133 low-risk pregnancies at the intra-abdominal portion, fetal end, and placental end of the cord. They found that the mean umbilical artery PI was higher at the intra-abdominal portion and fetal end than at the placental end of the cord ${ }^{[3]}$.

Khare et al. (2006) conducted a cross-sectional study on 71 pregnant women with singleton fetuses at 24-38 weeks of gestation. They recorded Doppler waves at the same four sites utilized in the present study. They reported that the highest mean values for PI were obtained from the PVC site, followed in a descending order by AI, FL, and PL sites ${ }^{[2]}$. 
Bhide et al. (2019) measured umbilical artery PI from the free loop of the cord and from the para-vesical site in 158 women with singleton pregnancy after 24 weeks of pregnancy. They stated that the umbilical artery PI at the para-vesical site was significantly higher than that in the free loop $(p<0.001)^{[12]}$.

This pattern of variation in PI among the different sites in the current study and in the literature can be attributed to a decreasing gradient across the length of the umbilical cord. Several theories were proposed to explain this difference in gradient which manifests from the fetal end up to the placental end of the umbilical cord. Trudinger (1987) attributed this difference to 'entrance region' phenomenon ${ }^{[13]}$. Another explanation was that the umbilical waveform may be reduced and weakened while propagating from the placental to the fetal ends. In addition, changes in the elasticity or the diameter of the umbilical vessel walls may account for the differences in the obtained Doppler velocity waveforms. This latter factor was suggested by Mine et al. (2001) who found that the RI of the umbilical artery decreased on the fetal side ${ }^{[14]}$.

Other factors seem also to affect the Doppler waveforms of the umbilical artery including gestational age $^{[15]}$, fetal heart rate and cardiac output ${ }^{[16]}$ and fetal blood viscosity ${ }^{[17]}$.

The variations in the umbilical artery PI among the different time points in the present study appear to be related to gestational age. In the present study, there was a significant, negative correlation between the umbilical artery PI (at each of FL, PL, PVC, and AI sites) and gestational age at the first visit. At the second and third visits, significant negative correlation with PI was found only at PVC site (visit 2) and AI site (visit 3). When the repeated measurements of umbilical artery PI were compared at each site, significant differences were detected among the three visits. The measurements, regardless of the site of sampling, were significantly lower at the third visit compared to the first and second visits. These findings suggest that standardization of the site of sampling may reduce variability and increase reliability of measurements. To confirm this inference, other prospective studies should assess this point after adjustment for other potential confounders such as gestational age, fetal heart rate, and laterality of the umbilical artery.

Sonesson et al. (1993) found that the difference in PI index between the fetal and placental sites was negatively correlated to gestational age $(\mathrm{r}=-0.25, p<0.001)^{[10]}$. Moreover, Bhide et al. (2019) reported that the umbilical artery PI significantly and negatively correlated with gestational age $(\mathrm{r}=-0.246$ at free loop site and -0.262 at the para-vesical site, $p<0.005)^{[12]}$.

The gestational age is considered as the major contributing factor to the variance recorded in the waveforms and measured indices of the umbilical artery using Doppler in a normal pregnancy population. This effect of gestational age could be attributed to the physiological changes which take place in the fetoplacental circulatory system during the progression of pregnancy. These physiological changes include both a progressive increase in the end-diastolic flow and a decrease in the flow pulsatility. Nevertheless, the variances in umbilical artery indices caused by differences in gestational age does not affect clinical practice where the umbilical artery indices are compared among patients with similar gestational age. The reference ranges for umbilical artery PI are constructed in relation to gestational age ${ }^{[18]}$.

It was observed in this study that the difference between measurements at PVC and AI sites became non-significant at the second and third visits. This finding is consistent with Acharya et al. (2005) who reported that the mean differences in umbilical artery PI between the fetal and placental ends decreased with progression of gestation ${ }^{[3]}$.

Other factors that may contribute to variability in blood flow velocity in the intra-abdominal umbilical artery include the filling of fetal urinary bladder and the umbilical cord coiling index ${ }^{[19]}$. However, these factors were not assessed in the current study.

The present study possessed several points of strength. First - being a longitudinal study - patients were assessed at different time points and at different gestational ages, allowing for comparisons of repeated measurements. Second, we included four sampling sites that are most adopted for measurement of umbilical artery waveforms, while most previous studies compared between two or three sites only. Third, we negated the effect of interobserver variability as all measurements were performed by the same observer.

On the other hand, this study was subject to some limitations. No distinction was made between the left or the right umbilical artery. The laterality of the umbilical artery may account for some variability of PI which was observed in our results, but we did not adjust for this confounding factor. Other confounding factors, such as the filling of fetal urinary bladder and the umbilical cord coiling index were not also assessed.

\section{CONCLUSION}

Standardization of the site of sampling of umbilical artery Doppler may reduce variability and increase reliability of measurements of umbilical artery PI as our study revealed significant variability in the measurements of umbilical artery PI among the 4 potential sampling sites as well as among the different time points at which the measurements were taken, with higher mean umbilical artery PI at PVC site, followed by AI, FL, and PL sites. with poor repeatability and reproducibility of the umbilical artery PI measurements. 


\section{CONFLICT OF INTEREST}

There are no conflicts of interests.

\section{REFERENCES}

1. Alfirevic, Z., Stampalija, T., \& Dowswell, T. (2017). Fetal and umbilical Doppler ultrasound in high-risk pregnancies. Cochrane database of systematic reviews, (6).1379-1387:172 .

2. Khare M, Paul S , et al. (2006): “Variation in Doppler indices along the length of the cord from the intraabdominal to the placental insertion". Acta obstetricia et gynecologica Scandinavica, 85(8): 922-928.

3. Acharya G, Wilsgaard T, Berntsen GKR, et al. (2005): ، ‘Reference ranges for serial measurements of blood velocity and pulsatility index at the intraabdominal portion, and fetal and placental ends of the umbilical artery". 26 (2): 162-169.

4. Maulik D, Mundy D, Heitmann E et al. (2010): Evidence-based approach to umbilical artery Doppler fetal surveillance in high-risk pregnancies: an update. Clin Obstet Gynecol. 2010;53 (4): 869-78.

5. Linde LE, Rasmussen S, Ebbing C, Baghestan E (2018): Placental abruption in parents who were born small: registry-based cohort study. BJOG: An International Journal of Obstetrics \& Gynaecology; 125(6):667-74.

6. Abramowicz JS, Warsof SL, Arrington J, et al. (1989): ،Doppler analysis of the umbilical artery. The importance of choosing the placental end of the cord". Journal of ultrasound in medicine : official journal of the American Institute of Ultrasound in Medicine; 8(4): 219-221.

7. Kay HH, Carroll BA, Bowie JD, et al. (1989): "Nonuniformity of fetal umbilical systolic/ diastolic ratios as determined with duplex Doppler sonography". Journal of ultrasound in medicine: official journal of the American Institute of Ultrasound in Medicine, 8(8): 417-420.

8. Vieyres P, Durand A, Patat F, et al. (1991): "Influence of the measurement location on the resistance index in the umbilical arteries: a hemodynamic approach". Journal of ultrasound in medicine : official journal of the American Institute of Ultrasound in Medicine, 10 (12): 671-675.

9. Mehalek KE, Rosenberg J, Berkowitz GS, et al. (1989): ،Umbilical and uterine artery flow velocity waveforms. Effect of the sampling site on Doppler ratios". Journal of ultrasound in medicine : official journal of the American Institute of Ultrasound in Medicine, 8(4): 171-176.

10. Sonesson SE, Fouron JC, Drblik SP, et al. (1993): “Reference values for Doppler velocimetric indices from the fetal and placental ends of the umbilical artery during normal pregnancy". Journal of clinical ultrasound: JCU, 21 (5): 317-324.

11. Schaefer M, Wittstock $G$ and Ville $Y$ (2000): “[Doppler ultrasound examination of fetal umbilical arteries of the intra-abdominal segment in normal singleton pregnancies]". Zeitschrift fur Geburtshilfe und Neonatologie, 204 (4): 135-139.

12. Bhide A, Badade A and Khatal K (2019): “The effect of sampling site on the variability of Umbilical artery PI". European Journal of Obstetrics \& Gynecology and Reproductive Biology, 235: 102-105.

13. Trudinger BJ (1987): “The umbilical circulation”. Semin Perinatol, 11 (4): 311-321.

14. Mine M, Nishio J, Nakai Y, et al. (2001): ، ‘Effects of umbilical arterial resistance on its arterial blood flow velocity waveforms". 80 (4): 307-310.

15. Maulik D, Yarlagadda AP, Youngblood JP, et al. (1989): “Components of variability of umbilical arterial Doppler velocimetry--a prospective analysis". American journal of obstetrics and gynecology, 160(6): 1406-1409.

16. Al-Gazali W, Chapman MG, Chita SK, et al. (1987): “Doppler assessment of umbilical artery blood flow for the prediction of outcome in fetal cardiac abnormality". British journal of obstetrics and gynaecology, 94(8): 742-745. 
17. Giles WB, Trudinger BJ, Paimer AA (1986): Umbilical cord whole blood viscosity and the umbilical artery flow velocity waveforms: a correlation. Br J Obstet Gynaecol; 93:466.

18. Akolekar R, Syngelaki A, Gallo DM, et al. (2015): “Umbilical and fetal middle cerebral artery Doppler at 35-37 weeks' gestation in the prediction of adverse perinatal outcome". Ultrasound in obstetrics \& gynecology: the official journal of the International Society of Ultrasound in Obstetrics and Gynecology, 46(1): 82-92.

19. Degani S, Lewinsky RM, Berger $\mathrm{H}$, et al. (1995): “Sonographic estimation of umbilical coiling index and correlation with Doppler flow characteristics". Obstetrics and gynecology, 86 (6): 990-993. 